Research Paper

\title{
DNA-dependent protein kinase catalytic subunit functions in metastasis and influences survival in advanced-stage laryngeal squamous cell carcinoma
}

\author{
Sha-sha He 1,3\#, Yong Chen 1,3\#, Xiao-ming Shen 1,6\#, Hong-zhi Wang7, Peng Sun1,5, Jun Dong 1,2, Gui-fang \\ Guo 1,2, Ju-gao Chen ${ }^{1}$, Liang-ping Xia 1,2, Pei-li Hu 1,2, Hui-juan Qiu 1,2, Shou-sheng Liu 1,2, Yi-xin Zhou ${ }^{1,2}$, \\ Wei Wang ${ }^{1,4}$, Wei-han $\mathrm{Hu}^{1,3}$, Xiu-yu Cai ${ }^{1,2 \bowtie}$
}

1. State Key Laboratory of Oncology in South China, Cancer Center, Sun Yat-Sen University, Guangzhou

2. $\quad$ Department of VIP Region, Cancer Center, Sun Yat-Sen University, Guangzhou, China

3. Department of Radiation, Cancer Center, Sun Yat-Sen University, Guangzhou, China

4. Department of Gastric Surgery, Cancer Center, Sun Yat-Sen University, Guangzhou, China

5. Department of Pathology, Cancer Center, Sun Yat-Sen University, Guangzhou, China

6. Department of Radiology, The First People's Hospital of Foshan (The affiliated Foshan Hospital of Sun Yat-Sen University), Foshan, Guangdong, China

7. Department of Radiation Oncology, Cancer Hospital, Chinese Academy of Medical Science, Peking Union Medical College, Beijing 100021, China

\#These authors have contributed equally to this work

$\triangle$ Corresponding authors: Wei-han Hu M.D., Ph.D. State Key Laboratory of Oncology in South China \& Department of Medical Oncology, Sun Yat-sen University Cancer Center, Guangzhou, 510060, China. Telephone: +0086-020-87343505, E-mail: huwh@sysucc.org.cn. Or Xiu-yu Cai M.D., Ph.D. State Key Laboratory of Oncology in South China \& Department of VIP Region, Sun Yat-sen University Cancer Center, Guangzhou, 510060, China. Telephone: +0086-020-87343498, E-mail: caixy@sysucc.org.cn

(C) Ivyspring International Publisher. This is an open access article distributed under the terms of the Creative Commons Attribution (CC BY-NC) license (https://creativecommons.org/licenses/by-nc/4.0/). See http://ivyspring.com/terms for full terms and conditions.

Received: 2017.03.14; Accepted: 2017.05.18; Published: 2017.07.22

\begin{abstract}
Background: DNA-dependent protein kinase catalytic subunit (DNA-PKcs) is known to function in several types of cancer. In this study, we investigated the expression and clinicopathologic significance of DNA-PKcs in laryngeal squamous cell carcinoma (LSCC).

Methods: We conducted a retrospective study of 208 patients with advanced-stage LSCC treated at Sun Yat-sen University Cancer Center, Guangzhou, China. We assessed DNA-PKcs and p16INK4a (p16) status using immunohistochemistry. We examined the association between DNA-PKcs expression and clinicopathologic features and survival outcomes. To evaluate the independent prognostic relevance of DNA-PKcs, we used univariate and multivariate Cox regression models. We estimated overall survival (OS) and distant metastasis-free survival (DMFS) using the Kaplan-Meier method.

Results: Immunohistochemical analyses revealed that 163/208 (78.4\%) of the LSCC tissue samples exhibited high DNA-PKcs expression. High DNA-PKcs expression was significantly associated with survival outcomes $(P=0.016)$ and distant metastasis $(P=0.02$; chi-squared test). High DNA-PKcs expression was associated with a significantly shorter OS and DMFS than low DNA-PKcs expression ( $P=0.029$ and 0.033 , respectively; log-rank test), and was associated with poor OS in the p16-positive subgroup $(P=0.047)$. Multivariate analysis identified DNA-PKcs as an independent prognostic indicator of OS and DMFS in all patients $(P=0.039$ and 0.037 , respectively).

Conclusions: Our results suggest that patients with LSCC in whom DNA-PKcs expression is elevated have a higher incidence of distant metastasis and a poorer prognosis. DNA-PKcs may represent a marker of tumor progression in patients with p16-positive LSCC.
\end{abstract}

Key words: Laryngeal squamous cell carcinoma; DNA-dependent protein kinase catalytic subunit (DNA-PKcs); p16INK4a (p16); prognosis 


\section{Background}

Laryngeal cancer is a prevalent malignancy, with an incidence of 3.5-5.5 per 100000 people and a mortality rate of 2.1-2.4 per 100000 people worldwide [1]. Laryngeal squamous cell carcinoma (LSCC) is the most common laryngeal cancer, accounting for approximately $90 \%$ of all malignant tumors of the larynx.

In clinical practice, treatment for LSCC is selected based on the TNM staging system because of the lack of an established prognostic model predicting clinical outcomes. In addition to clinical and pathologic grading, several biomarkers with prognostic value for clinical outcomes have been identified in laryngeal tumors. Recently, human papillomavirus (HPV) infection has gained attention for its role in the carcinogenesis of head and neck squamous cell carcinoma (HNSCC). HPV is found in $25.9 \%$ of all HNSCCs and $24 \%$ of laryngeal carcinomas [2], and emerging evidence has identified HPV-associated HNSCC as a separate subgroup of head and neck cancers with a distinct epidemiology, set of histopathologic characteristics, therapeutic response to chemotherapy and radiotherapy, and clinical outcome [3]. Compared with patients with HPV-negative HNSCC, patients with HPV-positive HNSCC exhibit a better response to induction chemotherapy and a concurrent chemoradiation protocol, and achieve a better overall outcome with lower mortality, metastasis, and recurrence rates [4-7]. Detection of HPV DNA is considered the gold standard for diagnosing HPV infection. However, carcinomas overexpressing p16INK4a (p16) likely represent tumors in which HPV was involved in the carcinogenic process [8]. p16, a cyclin-dependent kinase inhibitor that prevents retinoblastoma phosphorylation and blocks cell-cycle progression at the G1-S checkpoint, is involved in HNSCC pathogenesis [9]. Although staining for p16 does not exactly match HPV DNA test results, some studies have reported that p16 overexpression is closely correlated with HPV infection in oropharyngeal squamous cell carcinomas (OPSCCs) [10]. Immunohistochemical staining for p16 not only represents HPV status, but also indicates the prognosis of HPV-associated OPSCCs [11], and p16 expression may even serve as a surrogate biomarker for HPV-associated OPSCCs [12].

DNA-dependent protein kinase is a serine/threonine protein kinase complex comprising the $\mathrm{Ku}$ heterodimer (Ku70/Ku80) and DNAdependent protein kinase catalytic subunit (DNAPKcs). It plays an important role in DNA damage repair and the maintenance of genomic stability [13], and functions in the repair of DNA double-strand breaks (DSBs) by non-homologous end joining (NHEJ) [14]. DNA-PKcs is a member of the phosphatidylinositol 3-kinase (PI3K) family, and a study have explored and illustrated the pathway of DNA-PKcs in the processes of proliferation, survival, apoptosis, migration, invasion, and cytoskeletal rearrangement [15]. DNA-PKcs expression has been shown to correlate with the response to therapy and clinical outcomes in various cancers, such as nasopharyngeal carcinoma [16], colorectal cancer [17], and esophageal cancer [18].

Several studies have suggested that HPV negatively regulates DNA-PKcs $[19,20]$. Thus, in this study, we investigated the prognostic value of DNA-PKcs expression in patients with LSCC. Furthermore, we explored the relationship between DNA-PKcs and p16 status and survival outcomes, and assessed the suitability of p16 status as a biomarker relative to HPV DNA detection.

\section{Methods}

\section{Patients and tissue specimens}

In this study, we examined paraffin-embedded LSCC tissue samples obtained from 208 patients with previously untreated, surgically resectable, advancedstage (Stages III and IV) LSCC. All patients were pathologically diagnosed and received surgery between May 1999 and December 2009 at Sun Yat-sen University Cancer Center (SYSUCC), Guangzhou, China. This study was approved by the Clinical Research Ethics Committee of SYSUCC; all participants provided written informed consent to participate prior to treatment.

\section{Immunohistochemistry}

We examined DNA-PKcs and p16 expression in the 208 human LSCC tissue samples using immunohistochemistry. The paraffin-embedded sections (4-um thick) were heated at $60^{\circ} \mathrm{C}$ for 2 hours and deparaffinized in xylene. Antigen retrieval was performed using EDTA (PH 8.0) in high-pressure steam four 5 minutes. Sections were incubated with $1 \%$ bovine serum Albumin to block nonspecific binding, and incubated with anti-p16 antibody (1:100 dilution; JC8, Santa Cruz, CA), or anti- DNA-PKcs antibody (1:400 dilution; Abcam, ab1832), and then the slides were placed in a moist chamber overnight at $4^{\circ} \mathrm{C}$. After washing, the tissue sections were treated with biotinylated anti-rabbit secondary antibody 
(Abcam), and horseradish peroxidase was subsequently applied. Finally, Meyer's hematoxylin was used for nuclear counterstaining.

All immunostained slides were judged by two independent pathologists without knowledge of the clinicopathologic information. DNA-PKcs expression in the cell nucleus was evaluated independently. The intensity of DNA-PKcs staining was categorized as follows: negative staining (score $=0$ ); weak staining (score $=1)$; moderate staining (score $=2$ ); or strong staining (score $=3$ ). The percentage of immunoreactive cells was also assessed. The percentage of cells expressing DNA-PKcs was classified as: $0 \%$ (score $=$ $0) ;<10 \%$ (score $=1) ; 11-50 \%$ (score $=2) ; 51-80 \%$ (score $=3$ ); or $>81 \%$ (score $=4$ ). The DNA-PKcs immunoreactive score for each tissue was calculated by multiplying the staining intensity score by the percentage of positive cells (to obtain values of $0,1,2$, $3,4,6,9$, or 12). In this study, the cutoff value for DNA-PKcs expression was defined on the basis of a receiver operating characteristic (ROC) curve. A score $>5$ was used to classify tumors with high expression, whereas a score $\leq 5$ was used to indicate low expression. p16 status was categorized as positive or negative according to whether p16 staining was present or absent.

\section{Clinical data}

Before treatment, all patients underwent a physical examination, routine hematologic testing, laryngoscopy, a pathologic assessment of biopsy tissue, magnetic resonance imaging of the head and neck, a chest X-ray, abdominal ultrasound, and an entire body bone scan, with or without positron -emission tomography-computed tomography. Overall stage was classified according to the seventh edition of the American Joint Committee on Cancer/Union for International Cancer Control staging system [21].

Medical records were collected retrospectively. Data on age, smoking status, alcohol consumption, familial history of cancer, tumor localization, pathologic type, histologic grade, overall stage, $\mathrm{T}$ category, $\mathrm{N}$ category, treatment, and survival and metastasis status were recorded.

No patients had a history of anticancer therapy, adjuvant or neo-adjuvant therapy, or other malignancies, and none had distant metastases at diagnosis. Patients in our study underwent laryngectomy when pathologically diagnosed.

\section{Follow-up and outcome}

Follow-up evaluations were performed every 3 months within the first 3 years, every 6 months for the following 2 years, and annually thereafter until death.
The last follow-up was December 2016; the median follow-up time was 94.6 (range: 10.3-212.2) months. Overall survival (OS) was defined as the time in months from the date of surgery until death from any cause within the follow-up period. Distant metastasisfree survival (DMFS) was measured from date of surgery to metastasis, death, or the last follow-up.

\section{Statistical analysis}

All statistical analyses were conducted using SPSS (version 22.0; IBM Corporation, Armonk, NY, USA). Pearson's $\chi 2$ and Fisher's exact tests were used to analyze the associations between DNA-PKcs expression and clinicopathologic features and clinical outcomes. DNA-PKcs scores were allocated into the high and low expression groups according to the optimal cutoff value determined by ROC curve analysis. Survival curves were calculated using the Kaplan-Meier method and compared using the log-rank test. Cox proportional hazards analysis was used for univariate and multivariate analyses to explore the effects of clinicopathologic variables and DNA-PKcs and p16 expression on survival. All parameters found to be significant in the univariate analysis were included in a multivariate survival analysis using the Cox regression model. A two-sided probability value $<0.05$ was considered statistically significant.

\section{Results}

\section{Immunohistochemical analysis of DNA-dependent protein kinase catalytic subunit and p16INK4a expression in laryngeal squamous cell carcinoma tissue samples}

In tissue samples from 208 patients with LSCC, DNA-PKcs immunostaining was primarily present in the cell nucleus. Overall, 163/208 (78.4\%) patients exhibited high expression in laryngeal tumor tissue, whereas 45/208 (21.6\%) patients exhibited low expression. p16 immunostaining in normal laryngeal epithelial and tumor cells was evident in the cytoplasm. Of the 208 patients, the laryngeal tumor tissue samples were p16-positive in $48(23.1 \%)$ and p16-negative in 160 (76.9\%). The representative figures were shown in Figure 1, about high and low expression of DNA-PKcs, positive and negative expression of $\mathrm{p} 16$.

\section{Correlations between DNA-dependent protein kinase catalytic subunit expression and clinicopathologic parameters}

Correlations between DNA-PKcs expression and various clinicopathologic parameters are listed in Table 1. DNA-PKcs expression was significantly related to survival outcomes $(P=0.016)$ and distant 


\section{p16 expression}

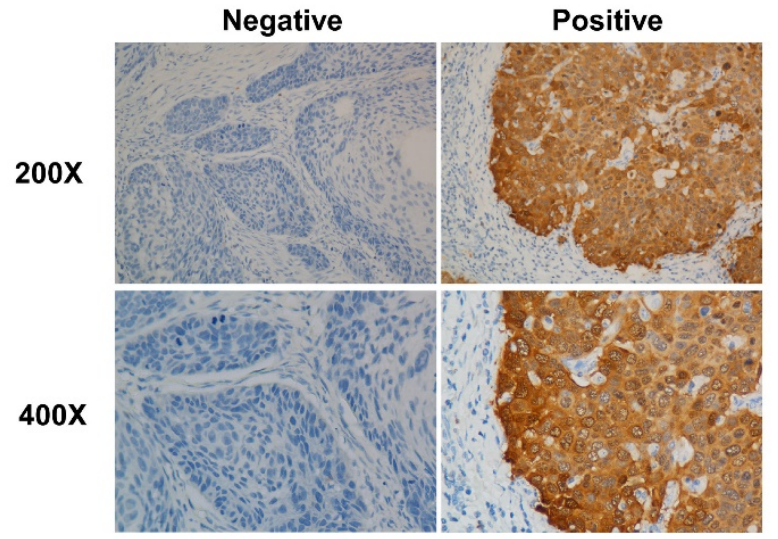

\section{DNA-PKcs expression}

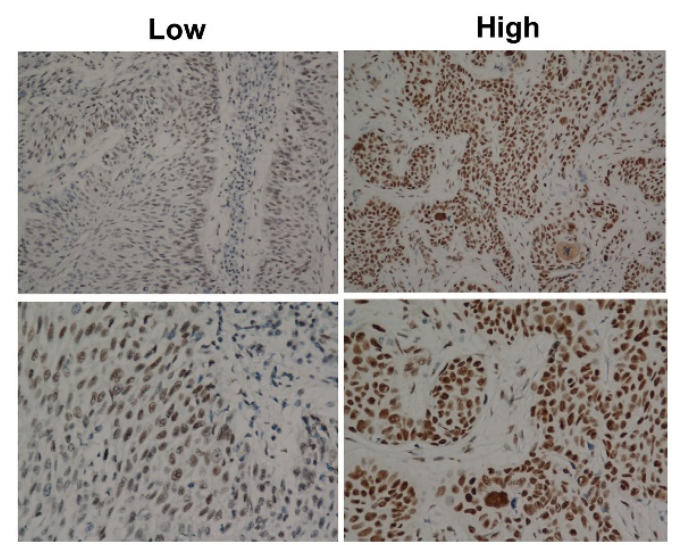

Figure 1. p16INK4A and DNA-PKcs overexpression in larynx tumors. Same region of same tumor staining positive for p16 (cytoplasm) and DNA-PKcs (nucleus), with magnification of $200 x$ and $400 x$.

metastasis status $(P=0.02)$. However, there was no statistically significant relationship between DNAPKcs expression and age, smoking status, alcohol consumption, tumor localization, histologic grade, lymph node metastasis, or p16 status.

Table 1. Correlations between the DNA-dependent protein kinase catalytic subunit expression and clinicopathologic characteristics of 208 patients.

\begin{tabular}{|c|c|c|c|c|c|}
\hline Characteristic & $\begin{array}{l}\text { Total } \\
(n, \%)\end{array}$ & $\begin{array}{l}\text { DNA-PKc } \\
\text { Low High }\end{array}$ & s expression & $x^{2}$ & $P$-value \\
\hline All cases & $208(100)$ & 45 (21.6) & $163(78.4)$ & & \\
\hline Age (years) & & & & 1.954 & 0.162 \\
\hline$<45$ & $107(51.4)$ & $19(42.2)$ & $88(54)$ & & \\
\hline$\geq 45$ & $101(48.6)$ & $26(57.8)$ & $75(46)$ & & \\
\hline Smoking & & & & 1.335 & 0.248 \\
\hline Yes & $184(88.5)$ & $42(93.3)$ & $142(87.1)$ & & \\
\hline No & $24(11.5)$ & $3(6.7)$ & $21(12.9)$ & & \\
\hline Alcohol consumption & & & & 2.64 & 0.104 \\
\hline Yes & $77(37)$ & $12(26.7)$ & 65 (39.9) & & \\
\hline No & $131(63)$ & $33(73.3)$ & $98(60.1)$ & & \\
\hline Localization & & & & 1.476 & 0.688 \\
\hline Supraglottic & $84(40.4)$ & $16(35.6)$ & $68(41.7)$ & & \\
\hline Glottic & $97(46.6)$ & $24(53.3)$ & $73(44.8)$ & & \\
\hline Subglottic & $9(4.3)$ & $1(2.2)$ & $8(4.9)$ & & \\
\hline Combination & $18(8.7)$ & $4(8.9)$ & $14(8.6)$ & & \\
\hline Histologic grade & & & & 0.124 & 0.94 \\
\hline Well & $73(35.1)$ & $15(33.3)$ & $58(35.5)$ & & \\
\hline Moderate & $101(48.6)$ & $22(48.9)$ & $79(48.5)$ & & \\
\hline Poor & $34(16.3)$ & $8(17.8)$ & $26(16)$ & & \\
\hline Lymph node metastasis & & & & 0.004 & 0.952 \\
\hline Negative & $128(61.5)$ & $28(62.2)$ & $100(61.7)$ & & \\
\hline Positive & $80(38.5)$ & $17(37.8)$ & $62(38.3)$ & & \\
\hline p16 status & & & & 0.306 & 0.58 \\
\hline Negative & $160(76.9)$ & $36(80)$ & $124(76.1)$ & & \\
\hline Positive & $48(23.1)$ & $9(20)$ & $39(23.9)$ & & \\
\hline Survival outcomes & & & & 5.816 & 0.016 \\
\hline Death & $93(44.7)$ & $13(28.9)$ & $80(49.1)$ & & \\
\hline Alive & $115(55.3)$ & $32(71.1)$ & $83(50.9)$ & & \\
\hline Distant metastasis & & & & 5.381 & 0.02 \\
\hline Yes & $36(17.3)$ & $13(28.9)$ & $23(14.1)$ & & \\
\hline No & $172(82.7)$ & $32(71.1)$ & $140(85.9)$ & & \\
\hline
\end{tabular}

Abbreviations: * chi-squared test.

\section{Univariate and multivariate analyses of overall survival}

The 5-year OS and DMFS rates for the entire cohort were $69.5 \%$ and $81.4 \%$, respectively. The cumulative 5-year OS and DMFS rates for patients with low DNA-PKcs expression were $84.7 \%$ and $88.7 \%$, respectively, compared with $65.7 \%$ and $78.9 \%$, respectively, for patients with high DNA-PKcs expression (log-rank test; $P=0.029$; Figure $2 \mathrm{~A}$ and $P=$ 0.033 ; Figure 2B). The 5 -year OS rate was $71.5 \%$ in the p16-negative group and $62.9 \%$ in the p16-positive group (log-rank test; $P=0.047$; Figure $2 C$ ). Univariate Cox regression analysis demonstrated that the clinical variables age at diagnosis, overall stage, lymph node metastasis, and p16 status were significantly associated with survival. A multivariate survival analysis indicated that high DNA-PKcs expression was an independent prognostic factor for OS (hazard ratio [HR]: $1.87,95 \%$ confidence interval [CI]: 1.199-2.763, $P$ $=0.039$ ) and DMFS (HR: 2.066, 95\% CI: 1.046-4.084, $P$ $=0.037$; Table 2 ).

\section{Subgroup analyses stratified by p16INK4a status}

Next, we stratified DNA-PKcs expression by p16 status and evaluated its prognostic value. Compared with patients with low DNA-PKcs expression, a trend toward shorter OS was observed in patients with high DNA-PKcs expression in the p16-positive subgroup $(P=0.037$; Figure 3A). Multivariate analysis further identified that DNA-PKcs was an independent indicator of OS in p16-positive patients (HR: 1.788, 95\% CI: 1.154-2.77, $P=0.034)$. However, this was not the case in p16-negative patients $(P=0.828$; Figure 3B). 

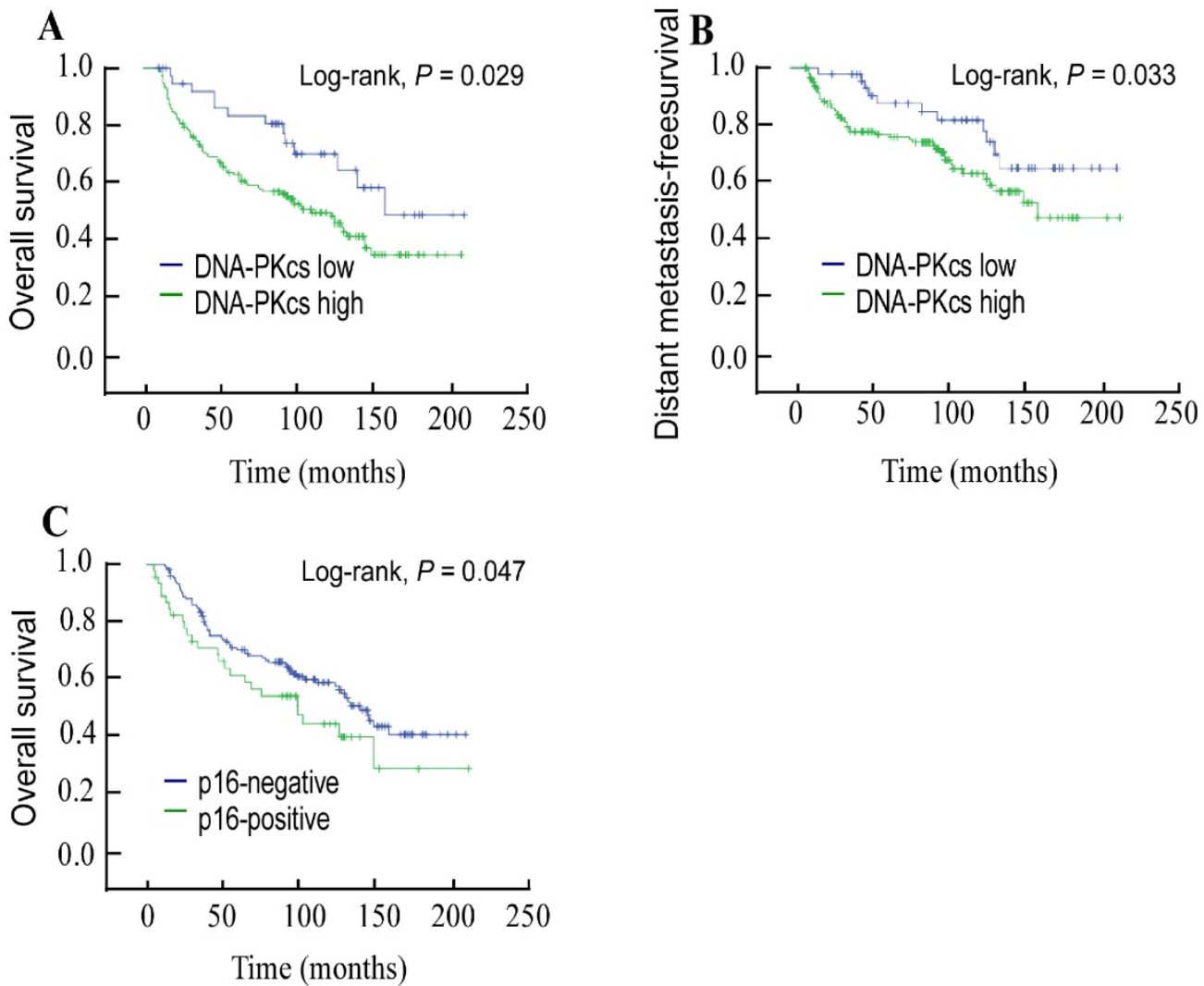

Figure 2. Kaplan-Meier overall survival (A) and distant metastasis-free survival (B) curves for 208 patients with laryngeal squamous cell carcinoma stratified by DNA-dependent protein kinase catalytic subunit expression; overall survival (C) stratified by p 161 NK4A status.

Table 2. Multivariate analysis of overall survival for 208 patients with laryngeal carcinoma.

\begin{tabular}{|c|c|c|c|c|}
\hline \multirow[t]{2}{*}{ Variable } & \multicolumn{2}{|l|}{$\begin{array}{l}\text { Overall } \\
\text { survival }\end{array}$} & \multicolumn{2}{|l|}{$\begin{array}{l}\text { Distant metastasis-free } \\
\text { survival }\end{array}$} \\
\hline & HR $(95 \%$ CI) & $P$ & HR $(95 \%$ CI) & $P$ \\
\hline \multicolumn{5}{|l|}{$\begin{array}{l}\text { Age at diagnosis } \\
\text { (years) }\end{array}$} \\
\hline$\geq 45$ vs. $<45$ & $\begin{array}{l}1.82 \\
(1.199-2.763)\end{array}$ & 0.005 & $2.044(1.035-4.037)$ & 0.039 \\
\hline \multicolumn{5}{|l|}{$\begin{array}{l}\text { Lymph node } \\
\text { metastasis }\end{array}$} \\
\hline $\begin{array}{l}\text { Positive vs. } \\
\text { negative }\end{array}$ & $\begin{array}{l}1.487 \\
(0.973-2.275)\end{array}$ & 0.067 & & \\
\hline \multicolumn{5}{|l|}{ Overall stage } \\
\hline IV vs. III & $\begin{array}{l}1.412 \\
(0.912-2.187)\end{array}$ & 0.122 & & \\
\hline \multicolumn{5}{|l|}{ DNA-PKcs } \\
\hline High vs. low & $\begin{array}{l}1.87 \\
(1.032-3.388)\end{array}$ & 0.039 & $2.066(1.046-4.084)$ & 0.037 \\
\hline \multicolumn{5}{|l|}{ p16 status } \\
\hline $\begin{array}{l}\text { Positive vs. } \\
\text { negative }\end{array}$ & $\begin{array}{l}1.782 \\
(1.009-3.146)\end{array}$ & 0.047 & $1.481(0.936-2.343)$ & 0.093 \\
\hline
\end{tabular}

\section{Discussion}

In this study, we showed that levels of DNA-PKcs and p16 expression were increased in primary LSCC tissues relative to adjacent noncancerous tissues. Patients with LSCC with high DNAPKcs expression experienced a shorter OS and DMFS than those with low DNA-PKcs expression, emphasizing the clinical value of DNA-PKcs expression in assessing the prognosis of patients with LSCC.

Emerging evidence has demonstrated that DNA-PKcs acts as a selective modulator to induce cell migration, invasion, and metastasis [22]. Studies have further identified DNA-PKCs as a modulator of cancer-associated pathways distinct from DNA repair, including the response to hypoxia, metabolic pathways, the inflammatory response, and transcriptional regulation. Meanwhile, clinical assessments have revealed that DNA-PKcs expression is significantly elevated in advanced disease, and is an independent predictor of metastases, recurrence, and reduced OS. Accordingly, an animal experiment demonstrated that silencing of DNA-PKcs suppresses tumor metastasis [23]. In our study, we identified DNA-PKcs as a marker of metastasis (HR for DMFS: 2.066, 95\% CI: $1.046-4.084, P=0.037)$ and survival prognosis (HR for OS: 1.87, 95\% CI: 1.199-2.763, $P=$ 0.039 ). Our findings are consistent with those of published studies that identify DNA-PKcs as associated with survival in various types of cancer [19, 24]. However, one study proposed that lower DNA-PKcs expression is correlated with a higher incidence of distant metastasis in patients with NPC [16]. The mechanisms underlying the functions of 

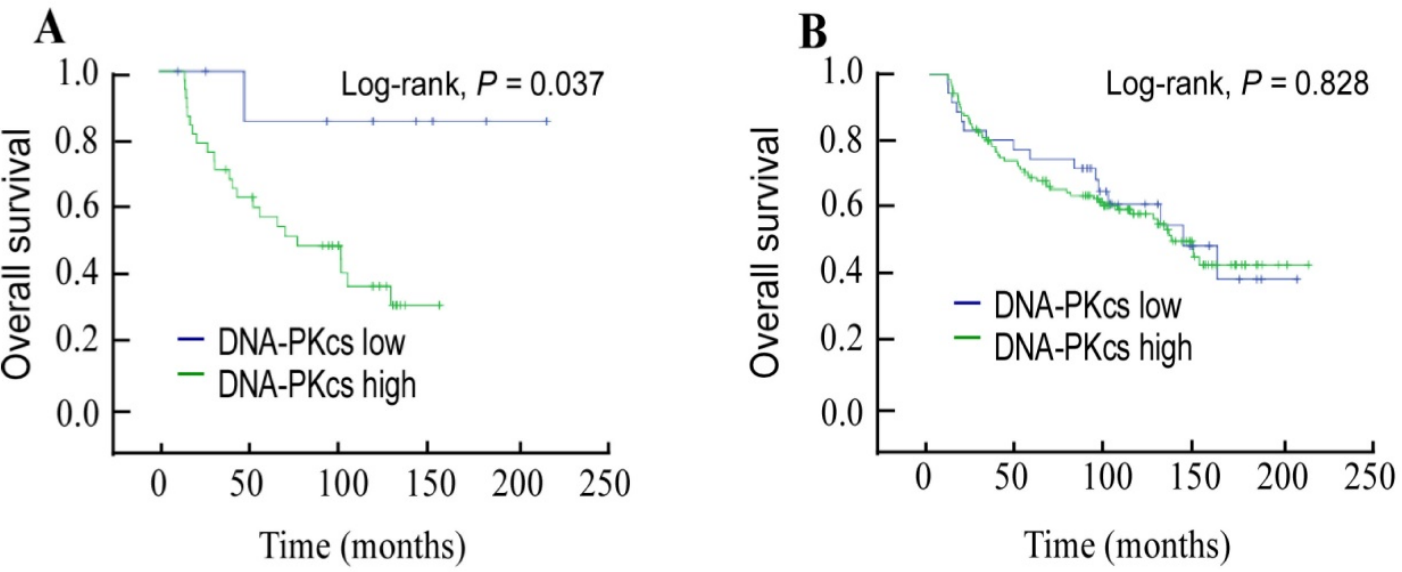

Figure 3. Kaplan-Meier overall survival curves for the subgroup of pl6INK4A-positive patients (A); and pl6INK4A -negative patients (B) stratified by DNA-dependent protein kinase catalytic subunit expression.

DNA-PKcs and p16 in tumors have yet to be elucidated; thus, further mechanistic studies are necessary to clarify the prognostic value of DNA-PKcs and p16 status in LSCC.

Increased DNA-PKcs activity may protect cancer cells from DNA-damaging agents such as chemotherapeutic drugs and radiation. Recently, lots of studies have explored a definite correlation between DNA-PKcs expression and the response of cancers to radio- and chemotherapy [25, 26]. DNA-PKcs, although best known for its role in NHEJ, has other roles in processes including the regulation of apoptosis, maintenance of telomere length, control of the cell cycle, and regulation of mitochondrial protein function [27]. It plays a crucial role in the repair of DSBs generated by cancer cures. However, the predicted association between DNA-PKcs expression and the response to treatment of human cancers is controversial and differs between stages and pathologic types. For example, studies have shown that inhibited the activity of DNA-PKcs may enhance the sensitivity of platinum compounds in the treatments of cancers including osteosarcoma and breast cancer [28, 29]. However, opposing results were obtained in breast cancer, where high DNA-PKcs expression was shown to predict a good response to radiotherapy [30]. Yet in lung carcinoma cell lines after irradiation, lower DNA-PKcs protein content and kinase activity were found in radiosensitive cells [31]. A recent study involving a small cohort of 29 patients found that DNA-PKcs knockdown increases the therapeutic sensitivity (to cisplatin and radiotherapy) and decreases the migration and invasion of HNSCC cells [19]. Together, these discoveries imply that DNA-PKcs has opposing roles in cancer, either favoring or inhibiting carcinogenesis and sensitizing or desensitizing cells to treatment methods depending on the cell context or tissue type [32]. However, in our study, we did not analyze the association between DNA-PKcs expression and therapeutic reactions. Thus, further, large -scale cohort studies are required to clarify the role of DNA-PKcs in the response to chemotherapy, radiotherapy, and other adjuvant therapies for LSCC.

In p16-positive tumors, the prognostic significance of DNA-PKcs for OS was retained (HR: 1.788, 95\% CI: $1.154-2.77, P=0.034)$. Despite limitations of our study related to the small number of patients involved, we believe that p16 may be complementary to DNA-PKcs and predict survival in patients with advanced-stage LSCC.

Interestingly, p16-positive patients exhibited a reduced OS compared with p16-negative patients $(P=$ 0.047). These findings are inconsistent with those of previous studies that concluded that HPV-positive patients experience a better response to treatment and overall outcome $[5,7]$. Thus, the interactions between p16 and HPV require further exploration. Another limitation of our study is that we did not examine HPV status: DNA-PKcs should be further studied as a potential marker of tumor progression in the presence of HPV and p16 expression.

\section{Conclusions}

In this study, we identified DNA-PKcs as a potent driver of tumor progression and metastasis, and highlighted the importance of DNA-PKcs as a potential marker for guiding adjuvant chemotherapy and radiotherapy in patients with LSCC. Future studies should explore the functional mechanisms and signaling pathways underlying DNA-PKcs activity and its influence on sensitivity to cancer treatment to enhance our understanding of the progression of this malignancy and improve clinical therapies. 


\section{Acknowledgments}

This work was supported by a grant from the Medical Research Foundation of Sun Yat-sen University of Guangdong Province, China [No. A2016011]. The funding agency had no role in the study design, data collection and analysis, decision to publish, or preparation of the manuscript.

\section{Competing Interests}

The authors declare that there are no conflicts of interest relevant to this article.

\section{References}

1. Jemal A, Bray F, Center MM, Ferlay J, Ward E, Forman D. Global cancer statistics. CA: a cancer journal for clinicians. 2011; 61: 69-90.

2. Kreimer AR, Clifford GM, Boyle P, Franceschi S. Human papillomavirus types in head and neck squamous cell carcinomas worldwide: a systematic review. Cancer epidemiology, biomarkers \& prevention: a publication of the American Association for Cancer Research, cosponsored by the American Society of Preventive Oncology. 2005; 14: 467-75.

3. Dok R, Nuyts S. HPV Positive Head and Neck Cancers: Molecular Pathogenesis and Evolving Treatment Strategies. Cancers. 2016; 8.

4. Ang KK, Harris J, Wheeler R, Weber R, Rosenthal DI, Nguyen-Tan PF, et al. Human papillomavirus and survival of patients with oropharyngeal cancer. The New England journal of medicine. 2010; 363: 24-35.

5. Dayyani F, Etzel CJ, Liu M, Ho CH, Lippman SM, Tsao AS. Meta-analysis of the impact of human papillomavirus (HPV) on cancer risk and overall survival in head and neck squamous cell carcinomas (HNSCC). Head \& neck oncology. 2010; 2: 15.

6. Charfi L, Jouffroy T, de Cremoux P, Le Peltier N, Thioux M, Freneaux P, et al. Two types of squamous cell carcinoma of the palatine tonsil characterized by distinct etiology, molecular features and outcome. Cancer letters. 2008; 260: 72-8.

7. Pannone G, Santoro A, Papagerakis S, Lo Muzio L, De Rosa G, Bufo P. The role of human papillomavirus in the pathogenesis of head \& neck squamous cell carcinoma: an overview. Infectious agents and cancer. 2011; 6: 4

8. von Knebel Doeberitz M. New markers for cervical dysplasia to visualise the genomic chaos created by aberrant oncogenic papillomavirus infections. European journal of cancer (Oxford, England: 1990). 2002; 38: 2229-42.

9. Zhang HS, Postigo AA, Dean DC. Active transcriptional repression by the Rb-E2F complex mediates G1 arrest triggered by p16INK4a, TGFbeta, and contact inhibition. Cell. 1999; 97: 53-61.

10. El-Naggar AK, Westra WH. p16 expression as a surrogate marker for HPV-related oropharyngeal carcinoma: a guide for interpretative relevance and consistency. Head \& neck. 2012; 34: 459-61.

11. Rischin D, Young RJ, Fisher R, Fox SB, Le QT, Peters LJ, et al. Prognostic significance of p16INK4A and human papillomavirus in patients with oropharyngeal cancer treated on TROG 02.02 phase III trial. Journal of clinical oncology: official journal of the American Society of Clinical Oncology. 2010; 28: 4142-8.

12. Reimers N, Kasper HU, Weissenborn SJ, Stutzer H, Preuss SF, Hoffmann TK, et al. Combined analysis of HPV-DNA, p16 and EGFR expression to predict prognosis in oropharyngeal cancer. International journal of cancer. 2007; 120: 1731-8.

13. Mahaney BL, Meek K, Lees-Miller SP. Repair of ionizing radiation-induced DNA double-strand breaks by non-homologous end-joining. The Biochemical journal. 2009; 417: 639-50.

14. Weterings E, Chen DJ. The endless tale of non-homologous end-joining. Cell research. 2008; 18: 114-24.

15. Kurimasa A, Ouyang H, Dong LJ, Wang S, Li X, Cordon-Cardo C, et al. Catalytic subunit of DNA-dependent protein kinase: impact on lymphocyte development and tumorigenesis. Proceedings of the National Academy of Sciences of the United States of America. 1999; 96: 1403-8.

16. Yang J, Xu X, Hao Y, Chen J, Lu H, Qin J, et al. Expression of DNA-PKcs and BRCA1 as prognostic indicators in nasopharyngeal carcinoma following intensity-modulated radiation therapy. Oncology letters. 2013; 5: 1199-204.

17. Hosoi Y, Watanabe T, Nakagawa K, Matsumoto Y, Enomoto A, Morita A, et al. Up-regulation of DNA-dependent protein kinase activity and Sp1 in colorectal cancer. International journal of oncology. 2004; 25: 461-8.

18. Tonotsuka N, Hosoi Y, Miyazaki S, Miyata G, Sugawara K, Mori T, et al. Heterogeneous expression of DNA-dependent protein kinase in esophageal cancer and normal epithelium. International journal of molecular medicine. 2006; 18: 441-7.

19. Weaver AN, Cooper TS, Wei S, Carroll WR, Rosenthal EL, Yang ES. DNA-PkCS expression in oropharyngeal squamous cell carcinoma: Correlations with human papillomavirus status and recurrence after transoral robotic surgery. Head \& neck. 2017; 39: 206-14.
20. Weaver AN, Cooper TS, Rodriguez M, Trummell HQ, Bonner JA, Rosenthal EL, et al. DNA double strand break repair defect and sensitivity to poly ADP-ribose polymerase (PARP) inhibition in human papillomavirus 16-positive head and neck squamous cell carcinoma. Oncotarget. 2015; 6: 26995-7007.

21. Edge SB, Compton CC. The American Joint Committee on Cancer: the 7th edition of the AJCC cancer staging manual and the future of TNM. Annals of surgical oncology. 2010; 17: 1471-4.

22. Goodwin JF, Knudsen KE. Beyond DNA repair: DNA-PK function in cancer. Cancer discovery. 2014; 4: 1126-39.

23. Goodwin JF, Kothari V, Drake JM, Zhao S, Dylgjeri E, Dean JL, et al. DNA-PKcs-Mediated Transcriptional Regulation Drives Prostate Cancer Progression and Metastasis. Cancer cell. 2015; 28: 97-113.

24. Zheng B, Mao JH, Li XQ, Oian L, Zhu H, Gu DH, et al. Over-expression of DNA-PKcs in renal cell carcinoma regulates mTORC2 activation, HIF-2alpha expression and cell proliferation. Scientific reports. 2016; 6: 29415.

25. Sun $X$, Yang $C$, Liu $\mathrm{H}$, Wang $\mathrm{O}, \mathrm{Wu} \mathrm{SX}, \mathrm{Li} X$, et al. Identification and characterization of a small inhibitory peptide that can target DNA-PKcs autophosphorylation and increase tumor radiosensitivity. International journal of radiation oncology, biology, physics. 2012; 84: 1212-9.

26. Tian X, Chen G, Xing H, Weng D, Guo Y, Ma D. The relationship between the down-regulation of DNA-PKcs or Ku70 and the chemosensitization in human cervical carcinoma cell line HeLa. Oncology reports. 2007; 18: 927-32.

27. Dip R, Naegeli $\mathrm{H}$. More than just strand breaks: the recognition of structural DNA discontinuities by DNA-dependent protein kinase catalytic subunit. FASEB journal: official publication of the Federation of American Societies for Experimental Biology. 2005; 19: 704-15.

28. Li X, Tian J, Bo Q, Li K, Wang H, Liu T, et al. Targeting DNA-PKcs increased anticancer drug sensitivity by suppressing DNA damage repair in osteosarcoma cell line MG63. Tumour biology: the journal of the International Society for Oncodevelopmental Biology and Medicine. 2015; 36: 9365-72.

29. Albarakati N, Abdel-Fatah TM, Doherty R, Russell R, Agarwal D, Moseley P, et al. Targeting BRCA1-BER deficient breast cancer by ATM or DNA-PKcs blockade either alone or in combination with cisplatin for personalized therapy. Molecular oncology. 2015; 9: 204-17.

30. Soderlund Leifler K, Queseth S, Fornander T, Askmalm MS. Low expression of Ku70/80, but high expression of DNA-PKcs, predict good response to radiotherapy in early breast cancer. International journal of oncology. 2010; 37: 1547-54.

31. O'Grady S, Finn SP, Cuffe S, Richard DJ, O'Byrne KJ, Barr MP. The role of DNA repair pathways in cisplatin resistant lung cancer. Cancer treatment reviews. 2014; 40: 1161-70.

32. Hsu FM, Zhang S, Chen BP. Role of DNA-dependent protein kinase catalytic subunit in cancer development and treatment. Translational cancer research. 2012; 1: 22-34. 